\title{
Introducción al análisis socio-ecológico a partir del mapeo colectivo del propio territorio: los usos por parte de distintos actores sociales y sus conflictos asociados
}

\section{Introduction to socio-ecological analysis based on the collective mapping of someone's own territory: uses made by different social actors and their associated conflicts}

\author{
Constanza Urdampilleta ${ }^{1}$ \\ https://orcid.org/0000-0001-5216-0768 \\ Carlos Ignacio Borón ${ }^{2}$ \\ http://orcid.org/0000-0003-3751-6724 \\ Sylvia Fischer ${ }^{3}$ \\ http://orcid.org/0000-0002-1184-0414 \\ Raúl Esteban Ithuralde ${ }^{4}$ \\ https://orcid.org/0000-0002-9757-0530
}

\begin{abstract}
Resumen: El presente trabajo expone una práctica de la materia "Ecología y Desarrollo" en el ámbito de una Facultad de ciencias naturales. El objetivo es fomentar la apropiación por parte del estudiantado del espacio del campus y de conceptos de conflictos socio-ambientales y sistemas socio-ecológicos. A través de un trabajo de debate y con el uso de imágenes aéreas, información bibliográfica, notas periodísticas, fotografías del lugar y el relato de un integrante del Vivero Comunitario Ciudad Universitaria, se reconstruyen históricamente los cambios en el paisaje y sus usos. Las dinámicas planteadas propiciaron la identificación de distintos actores involucrados, visualización espacial de los usos que estos hacen del espacio, y la identificación de diferentes conflictos. Esta herramienta didáctica y técnica resultó un dispositivo valioso para facilitar el diálogo entre distintos actores.
\end{abstract}

Palabras clave: Educación superior. Ecología social. Interacción ecológica. Campus universitario. Conciencia ecológica.

\begin{abstract}
The present article exposes a practice in the course "Ecology and Development" in a school of natural sciences. The objective is to promote the students' appropriation of the campus space, the socio-environmental conflicts, and the concepts of socio-ecological systems. Through discussion and the use of aerial images, bibliographical information, journalistic notes, photographs and the narration of a member of the University Campus Community Plant Nursery, landscape changes and its uses are historically reconstructed. The work has led to the identification of different actors involved, a spatial visualization of the uses they make of space, and the identification of conflicts. This didactic and technical tool has proven to be a valuable device to facilitate dialogue among different actors.
\end{abstract}

Keywords: Higher education. Social ecology. Ecological interaction. University campus. Ecological consciousness.

\footnotetext{
${ }^{1}$ Universidad de Buenos Aires (UBA), Facultad de Arquitectura Diseño y Urbanismo, Buenos Aires, Argentina. E-mail: coniurdampilleta@gmail.com

${ }^{2}$ Universidad Nacional de San Martín, Instituto de Investigación e Ingeniería Ambiental, Instituto de Nanosistemas (INS), San Martín, Buenos Aires, Argentina.

${ }^{3}$ UBA, Facultad de Ciencias Exactas y Naturales, Departamento de Ecología, Genética y Evolución, e IEGEBA (UBA-Conicet), Buenos Aires, Argentina.

${ }^{4}$ Universidad Nacional de Santiago del Estero, Instituto de Estudios para el Desarrollo Social, Facultad de Humanidades y Ciencias Sociales y de la Salud, Santiago del Estero, Santiago del Estero, Argentina.
} 


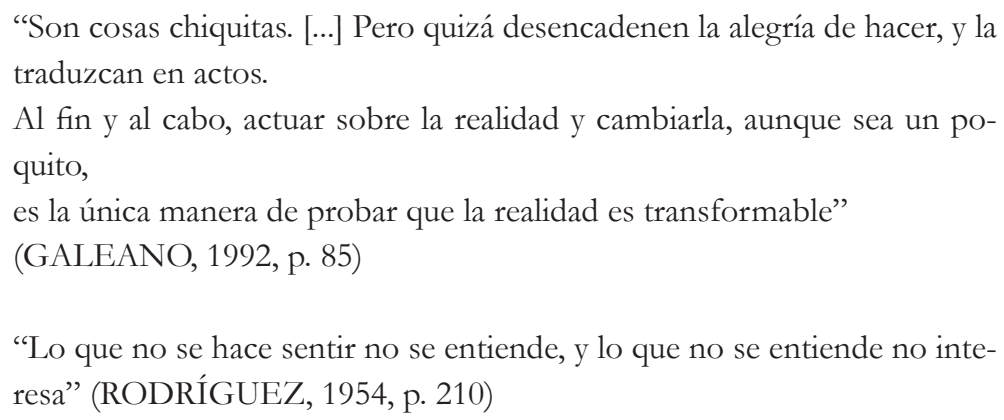

\section{Introducción}

La educación en la carrera de Ciencias Biológicas de la Facultad de Ciencias Exactas y Naturales (FCEN) de la Universidad de Buenos Aires (UBA) está focalizada ante todo en aspectos técnicos de la disciplina, pensada mayormente para formar a profesionales que se desempeñarán en el ámbito académico. Pocas materias incluyen contenidos orientados al desarrollo profesional del biólogo fuera del ámbito académico, como tampoco de reflexión sobre la práctica profesional en sus implicancias éticas, políticas, económicas y culturales. En el área de Ecología, la materia Ecología y Desarrollo tiene como objetivo principal el análisis de relaciones entre el mundo social y el mundo natural (UNIVERSIDAD DE BUENOS AIRES, 2017). En este marco, hemos introducido un trabajo práctico de mapeo colectivo de actores y sus diversas y contrapuestas visiones, intereses y usos de los espacios y los conflictos ambientales construidos en el territorio de Ciudad Universitaria (CU) de la UBA, del que forman parte estudiantes y docentes involucrados.

Entendemos que el objeto de aprendizaje son, en grandes líneas, los territorios (FERNANDES, 2009). No podemos entender al ambiente y las relaciones ecológicas (que serían el objeto de aprendizaje clásico de una materia de ecología en la carrera de Ciencias Biológicas) por fuera del sistema de relaciones sociales entre las personas que hoy conforman al sistema-mundo capitalista, menos aún considerando el carácter extractivista del mismo, presente desde su génesis (HARVEY, 2004; QUIJANO; WALLERSTEIN, 1992). Desde este punto de vista, todo conflicto ambiental es siempre un conflicto social, enmarcado en distintas representaciones y disputas por usos de los recursos y el territorio y, entonces, en la lucha de clases.

El campo de la Educación en Ambiente ha desarrollado sucesivos abordajes para comprender y proponer situaciones de aprendizaje de estos sistemas complejos (GARCÍA, 1999) desde una pedagogía crítica (LEFF, 1999). A su vez, algunos autores y autoras del movimiento Ciencia, Tecnología y Sociedad (CTS) desde una visión crítica y social de las ciencias han incorporado también la perspectiva ambiental para integrar un enfoque Ciencia, Tecnología, Sociedad y Ambiente (ACEVEDO-DÍAZ; VÁZQUEZ ALONSO; MANASSERO MAS, 2009). En palabras de Enrique Leff:

La complejidad ambiental irrumpe en el mundo como un efecto de las formas de conocimiento, pero no es solamente una relación de conocimiento. 
No es una biología del conocimiento ni una relación entre el organismo y su medio ambiente. La complejidad ambiental no emerge de las relaciones ecológicas, sino del mundo tocado y trastocado por la ciencia, por un conocimiento objetivo, fragmentado, especializado. No es casual que el pensamiento complejo, las teorías de sistemas y las ciencias de la complejidad surjan al mismo tiempo que se hace manifiesta la crisis ambiental, allá en los años sesenta, pues el fraccionamiento del conocimiento y la destrucción ecológica son síntomas del mismo mal civilizatorio. Por ello, la complejidad ambiental remite a un saber sobre las formas de apropiación del mundo y de la naturaleza a través de las relaciones de poder que se han inscrito en las formas dominantes de conocimiento. Desde allí se abre el camino que hemos seguido por los senderos de este territorio desterrado de las ciencias, para delinear, comprender y dar su lugar - su nombre propio - a la complejidad ambiental (LEFF, 2007, p. 11).

Es desde esta perspectiva que nos posicionamos al plantear una práctica en la materia Ecología y Desarrollo que proponga al estudiantado el análisis de su propio territorio, el de la Ciudad Universitaria de la Universidad de Buenos Aires, a través de la narrativa de actores involucrados, material periodístico, el uso y la construcción de mapas y de herramientas de la cartografía crítica. De esta forma, también se hacen cargo de analizar los propios conflictos ambientales que atraviesan a la institución, al estudiantado y al cuerpo docente como sujetos que habitan, usan y disputan ese espacio. Esta práctica habilita a un poner el cuerpo que es infrecuente en la formación de futuros biólogos y biólogas. Esto permite, aun de forma incipiente, dialogar con otros saberes como parte de un trabajo curricular de la carrera de Ciencias Biológicas, que para muchos y muchas estudiantes será la primera vez en realizar esta tarea como parte de un trabajo en una asignatura de la carrera. Buscamos visibilizar los conflictos de intereses como algo inherente a la territorialización y el uso del espacio, situándonos en el mismo espacio que habitamos como universitarios y universitarias para desde allí emprender la enseñanza y el aprendizaje (MASSARINI; SCHNEK, 2015).

\section{La carrera de Ciencias Biológicas y la Materia de Ecología y Desarrollo}

La materia Ecología y Desarrollo está planteada como materia opcional en la carrera de Licenciatura en Ciencias Biológicas. Esta carrera consta de tres ciclos: un Ciclo Básico Común (de un año de duración, y gestionado por la Universidad de manera directa), un Ciclo Básico (de trece materias obligatorias, aproximadamente tres años y medio) y un Ciclo Superior Orientado (diez materias o nueve materias y una Tesina de Licenciatura). Para cursar el Ciclo Superior debe firmarse un Plan Individual de Estudios, acordado con un tutor y refrendado por la Comisión de Carrera en Ciencias Biológicas, que en la orientación de Ecología consta de una materia de Evolución, una materia de Fisiología (a elegir entre tres opciones), dos materias troncales (a elegir entre tres materias de Ecología), dos materias recomendadas (para las cuales hay ocho opciones y donde se encuentra Ecología y Desarrollo), y además debe cursarse una materia taxonómica. Las restantes materias pueden acordarse con el tutor en base a una lista amplia de optativas (más de 50). 
Las materias obligatorias y troncales para la orientación de Biología analizan los ecosistemas y los territorios sin considerar las dinámicas sociales allí presentes, salvo en algunos casos como un apartado especial. En este sentido, la materia Ecología y Desarrollo viene a poner en el centro de análisis a los sujetos, la sociedad y sus relaciones con la naturaleza. La materia tiene como objetivo el estudio de la interacción humana con los ecosistemas, analizando los cambios antropogénicos y las medidas para disminuir el impacto ambiental. Los ejes de la materia son: Sustentabilidad, Ejes y Servicios Ecosistémicos, Factores Antropogénicos de Cambio Ambiental, respuesta a cambios Antropogénicos, Agua, Alimento, Energía, Minería, Residuos, Salud y Ambiente, Restauración de Ecosistemas, Bioeconomía, Huella Ecológica, Legislación Ambiental, Evaluación del Impacto Ambiental, Responsabilidad Social Empresarial, Inequidad, Pobreza y Ambiente (UNIVERSIDAD DE BUENOS AIRES, 2017). Este Trabajo Práctico busca poner de manifiesto la existencia de distintos actores en el territorio que han construido diversas apropiaciones del mismo y portan cosmovisiones sobre el ambiente a veces contradictorias entre sí. Se propone reflexionar sobre la información que brindan los mapas en tanto representaciones (sociales) del espacio y de sus cargas ideológicas y de intereses, como así también identificar distintas áreas de acuerdo a su uso y las disputas presentes (por los recursos, servicios ecosistémicos, valoraciones, cosmovisiones ambientales y de vida). Se necesita entonces trabajar profundamente sobre ciertas herramientas de análisis de la realidad social y natural, que puedan ser utilizadas para alcanzar una comprensión más amplia de ecosistemas altamente antropizados (como ecosistemas urbanos, agroecosistemas modificados por polución, etc.).

\section{El territorio en cuestión}

La Ciudad Universitaria de la UBA está ubicada en la porción Norte del área costera de la Ciudad Autónoma de Buenos Aires sobre el Río de la Plata, en una franja de terreno de 130 hectáreas de forma irregular. Gran parte de esta superficie son terrenos ganados al río mediante relleno con escombros durante las décadas de 1960 y 1970, que quedaron abandonados luego de la paralización del proyecto durante la última dictadura (1976-1983). Llegaron a construirse 3 edificios, Pabellones (I; II; III), y quedaron los cimientos de dos más (IV; V). Durante las últimas décadas, el área de los cimientos abandonados y de relleno fue progresivamente ocupada por una densa vegetación silvestre mediante la hidrodinámica del río y sus afluentes. Se puede observar la distribución de los espacios en la Figura 1. De igual manera, el área fue ocupada por asentamientos de personas en distintas circunstancias. Estos eran percibidos como conflictivos y consecuentemente ocurrieron desalojos en 1998 y 2006. En 2007 se formó la eco-aldea Velatropa, en los cimientos abandonados del Pabellón V. Velatropa se crea con una filosofía que replantea la relación cultura-naturaleza; es posible que esto llevara a una convivencia relativamente tolerada por las autoridades de la Universidad de Buenos Aires (CENTRO INTERDISCIPLINARIO DE EXPERIMENTACIÓN Y EDUCACIÓN AMBIENTAL, 2017). En 2015, en consonancia con Velatropa, se inicia Vivero Comunitario Ciudad Universitaria (ViCCU), un vivero de plantas nativas, cuyos integrantes plantean además la gestión comunitaria del espacio verde de Ciudad Universitaria. 
En parte de los terrenos rellenados y posteriormente ocupados por vegetación se ubica la Reserva Ecológica Costanera Norte: corresponde a una superficie de 18 ha con una diversidad de unas 400 especies de animales y vegetales. Su origen legal comienza en agosto de 2000, cuando la Universidad de Buenos Aires y el Gobierno de la Ciudad de Buenos Aires comenzaron a articular un proyecto de Parque Natural. En 2006 comienza el movimiento de tierra en manos del Gobierno de la Ciudad y en 2010 se presenta un proyecto de ley para declararla Reserva Ecológica. En ambos casos hubo conflictos por la falta de diálogo y previo consentimiento entre distintas partes. El 14 de diciembre de 2012 se sanciona la ley 4467, a partir de un proyecto que contempla cada uno de los requerimientos expresados por el Consejo Directivo de la Facultad de Ciencias Exactas y Naturales, se crea la "Reserva Ecológica Costanera Norte" (al día de hoy no se firmó el convenio necesario) (COARECN, 2015).

Paralelamente, a principios de 2015 el Gobierno de la Ciudad de Buenos Aires y el Rectorado de la Universidad hacen un convenio de "Renovación total de espacios de tránsito y de transporte público y privado". El mismo resultó conflictivo en un principio por la falta de conocimiento de la comunidad del plan de obras, manifestándose incluso el Consejo Directivo de la FCEN al respecto, mediante resolución 141/2015 (UNIVERSIDAD DE BUENOS AIRES, 2015). En los planos puede observarse que se pretendía avanzar sobre el espacio correspondiente a Velatropa y ViCCU. Sin embargo el conflicto se desata previamente a tener acceso a los planos, durante febrero de 2015, cuando topadoras avanzan sobre el espacio entre los cimientos de los pabellones IV y V. La resistencia de los y las integrantes de la comunidad Velatropa hizo que se frenara el avance en ese momento, pero la tensión permanece latente.

Las dificultades para poner en funcionamiento el espacio de Reserva y el comienzo sin consulta previa de obras de infraestructura sobre el espacio verde público revelaron la necesidad de generar mayor participación de la comunidad en su planificación y gestión.

Es en este contexto que se creó una propuesta didáctica de un trabajo práctico para la materia Ecología y Desarrollo de la FCEN-UBA, que tuvo su primera experiencia en el año 2016, con el objetivo de fomentar la apropiación por parte del estudiantado del espacio del campus; y el trabajo con conceptos de conflictos ambientales, sistemas socio-ecológicos y ecología de paisajes.

\section{Esquema del trabajo práctico}

\section{Objetivos}

El objetivo general de la práctica es fomentar la apropiación por parte del estudiantado del espacio del campus; y el trabajo con conceptos de conflictos socio-ambientales, sistemas socio-ecológicos y ecología de paisajes.

Los objetivos particulares que se desprenden son:

a) generar un espacio de diálogo y problematización de la gestión de CU;

b) identificar actores presentes en $\mathrm{CU}$;

c) mapear participativamente el uso de CU que hace cada actor identificado;

d) generar un mapa unificado del uso que los distintos actores hacen de CU;

e) identificar conflictos que surgen en la utilización y apropiación del espacio de CU. 


\section{Contenido}

Cartografía crítica: Los mapas son modelos abstractos efectivos para la trasmisión de información espacial. Históricamente la cartografía fue utilizada como herramienta exclusiva del Estado y de otras autoridades externas. Al ser aceptados como representaciones naturales e incuestionables, estas cartografías han sido funcionales a la imposición de ordenamientos territoriales y a la planificación de la apropiación de los servicios ecosistémicos (RISLER; ARES, 2014; SLETTO et al., 2013). La cartografía crítica nos provee herramientas conceptuales para deconstruir la asimilación del mapa hegemónico partiendo de que el mapa no es el territorio - que representa -, repensar críticamente la carga ideológica y contextos sociales que hay detrás de ellos y reflexionar sobre la ideología presente en la construcción de los mismos y reconceptualizar la manera en que personalmente lo hacemos.

Mapeo participativo: la herramienta de mapeo participativo, generada dentro del ámbito conceptual de la cartografía crítica, propone la democratización de la producción de mapas, incentivando a los actores locales a producirlos, siendo que éstos poseen un conocimiento profundo y rico del territorio, para dar cuenta de sus representaciones plurales y colectivas. Esta práctica genera instancias de intercambio colectivo que permiten visualizar el territorio desde nuestra mirada, reflexionar colectivamente sobre los problemas, disputas y relaciones de poder en un territorio particular; y facilita el abordaje y la problematización de territorios sociales, subjetivos, geográficos. Esta práctica puede ser pensada como un proceso social que sirve para fortalecer otros procesos educativos, identitarios y organizacionales. Es pertinente señalar que los mapas son acotados, muestran algunos aspectos de la realidad, simplificaciones (RISLER; ARES, 2014; SLET'TO et al., 2013).

Identificación de actores claves: Otra herramienta de análisis fundamental para abordar el entendimiento del territorio es la conceptualización e identificación de los actores involucrados (sus intereses, motivos, estrategias de acción y conflictos con respecto al uso de recursos) (BRENNER, 2010). Los actores pueden ser individuos o colectivos que actúan de formas específicas según sus propios intereses, llevan a cabo diversas acciones para asegurarse el acceso y/o control sobre ciertos bienes comunes existentes en un lugar o región. Se los identifica y define según el caso y objetivo de análisis en relación a un eje específico (TAPELLA, 2007).

\section{Dinámica}

En el cuadro 1 se presenta la dinámica planificada para el taller. Se propone disponer del tiempo para la instancia de mapeo y puesta en común priorizando la participación e intercambio entre las y los estudiantes. 
Introducción al análisis socio-ecológico a partir del mapeo ...

Cuadro 1 - Dinámica planificada para el taller participativo de mapeo de usos de CU por distintos actores e identificación de conflictos

\begin{tabular}{|c|c|c|c|}
\hline Sección & Objetivo & Actividad & Tiempo \\
\hline 0) Presentación & Justificación del trabajo práctico & Exposición docente & $20 \mathrm{~min}$ \\
\hline 1) Introducción & $\begin{array}{l}\text { Introducción teórica y reconstrucción } \\
\text { de la historia reciente del campus }\end{array}$ & Exposición docente & 1 hora \\
\hline \multirow{2}{*}{$\begin{array}{l}\text { 2) Mapeo del } \\
\text { propio territorio }\end{array}$} & a) Identificación de actores & Lluvia de ideas & $15 \mathrm{~min}$ \\
\hline & $\begin{array}{l}\text { b) Mapeo de usos del espacio por } \\
\text { distintos actores }\end{array}$ & Trabajo en grupos & $45 \mathrm{~min}$ \\
\hline $\begin{array}{l}\text { 3) Puesta en } \\
\text { común y discusión }\end{array}$ & $\begin{array}{l}\text { Puesta en común, identificación de } \\
\text { conflictos y problematización }\end{array}$ & $\begin{array}{l}\text { Exposición de los grupos } \\
\text { con lugar a discusión }\end{array}$ & $\begin{array}{l}1 \text { hora, } 30 \\
\text { min }\end{array}$ \\
\hline 4) Cierre & Recopilación del trabajo & Exposición docente & $10 \min$ \\
\hline \multicolumn{3}{|l|}{ Total } & 4 horas \\
\hline
\end{tabular}

Fuente: elaboración de los autores.

\section{Breve introducción teórica y reconstrucción de la historia reciente (a cargo de los docentes y de actores de la CU, del ViCCU)}

Se comienza haciendo foco en el territorio de estudio, realizando una breve construcción de la historia reciente del espacio para contextualizar. Se parte de un mapa de Ciudad Autónoma de Buenos Aires (C.A.B.A.) de 1921 y fotografías aéreas de 1964, 1978 y 1998 para mostrar la evolución costera producto de la variación de las geoformas (relleno con escombros) y su relación con la hidrodinámica del estuario, tomado de Marcomini y López (2004). Esto se complementa con información bibliográfica sobre la historia de construcción del campus. Además se usan artículos periodísticos para mostrar la existencia de asentamientos de viviendas informales que fueron desalojados en 1998 y 2006, y la percepción de los mismos. Finalmente se cuenta con el relato de un vecino, integrante de ViCCU, sobre la historia de uso de los espacios verdes: los asentamientos informales, la conformación de la eco-aldea Velatropa y el vivero de plantas nativas, ViCCU.

En ese contexto se propone tomar las herramientas de la cartografía crítica, el mapeo participativo y la identificación de actores involucrados para aproximarse al entendimiento crítico del territorio en cuestión. Entonces se da una breve introducción teórica planteando preguntas respecto a la cartografía hegemónica y la carga ideológica de los mapas, usando como soporte la arbitrariedad de la posición del Norte hacia arriba en los mapas y la comparación entre dos proyecciones diferentes del planisferio (Mercator versus Gall-Peters), señalando la posición de la línea del ecuador para mostrar la distorsión que impone la primera proyección. Desde el interés en construir una visión crítica del territorio, se propone la herramienta de mapeo participativo para reflexionar colectivamente sobre el área de estudio, los actores involucrados, sus intereses y su uso de los espacios.

\section{Identificación de actores}

A partir de una lluvia de ideas se realiza un listado de los diferentes actores que hacen uso de CU. Se clasifica por grupos de actores según similitudes en relación al uso del espacio (TAPELLA, 2007). 


\section{Mapeo participativo}

Para el mapeo participativo se usa como base el Manual de mapeo colectivo (RISLER; ARES, 2013). El foco de realizarlo diferenciando actores surge a partir de la propuesta teórica y metodológica de Díaz et al. (2011). En el taller se disponen mapas soporte y acetatos con esquema del campus (sin referencias), que se presentan en la Figura 1; marcadores de colores indelebles, alcohol, algodón, reglas, y una propuesta de pictogramas adaptada al caso. Haciendo uso de estos elementos, la consigna es, en grupos, explicitar espacialmente los intereses y los usos del espacio personificados por cada uno de los actores identificados (en esta instancia no se pone el foco en los conflictos).

\section{Puesta en común y discusión}

Con un retroproyector se proyectan los mapas por actores realizados en los grupos y se elabora un mapa unificado en papel afiche con todos los actores, utilizando colores distintos para cada uno.

Esta instancia se propone como una instancia de síntesis, en la cual se reúnen los distintos actores mapeados sobre el territorio común. Se da lugar para problematizar la interacción entre los actores y a la identificación de disputas y conflictos.

Figura 1A - Fotografía aérea del campus usada como mapa de soporte; Figura 1B - Esquema del campus con referencias
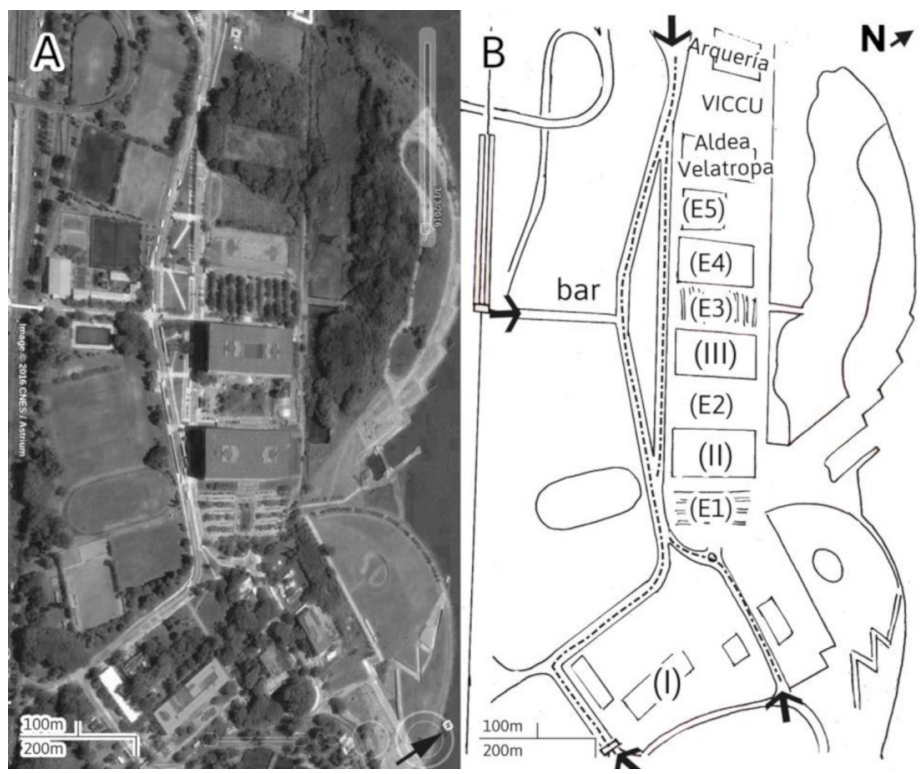

En 1B las flechas indican los accesos, las líneas punteadas el tránsito vehicular, (I), (II) y (III) son los pabellones donde se dictan clases, (E1-5) son los estacionamientos.

Fuentes: (1A) Google earth (2018) y (1B) Elaborado por los autores. 


\section{Validación del trabajo práctico}

Este artículo es una sistematización de los trabajos prácticos realizados en la materia Ecología y Desarrollo en los años 2016, 2017 y 2018. Recurrimos a la sistematización de experiencias como herramienta metodológica para recuperar y evaluar experiencias pasadas significativas, produciendo en el proceso un nuevo conocimiento a partir del diálogo y la reflexión colectiva (CENDALES GONZÁLEZ; TORRES CARRILLO, 2006). Recogemos información que pueda complementar a nuestras memorias y que las interpele, como: (a) audios de los trabajos prácticos; (b) planificaciones de los trabajos prácticos; (c) informes del estudiantado de los trabajos prácticos; (d) mapas construidos por los grupos de estudiantes; (e) entrevistas a docentes; (f) encuestas digitales con preguntas estructuradas y semiestructuradas a estudiantes participantes. Para realizar este trabajo construimos ejes-problemas que interrogan a la experiencia (ZEMMELMAN, 2011): (a) la amplitud del mapeo de actores por parte de los y las estudiantes; (b) el uso dado a los mapas por el estudiantado; (c) la identificación de conflictos entre actores y qué tipos de conflictos son identificados en cada momento del taller; (d) las discusiones y preguntas propuestas por las y los estudiantes; (e) el grado de aceptación del TP por estudiantes y docentes. A partir del análisis de estos ejes-problema proponemos nuevas modificaciones a la planificación.

\section{Resultados y discusión}

En las primeras experiencias del taller se logró una amplia y activa participación, permitiendo un rico intercambio de experiencias y visiones entre estudiantes, docentes y expositores. En los tres años hubo estudiantes que participan en grupos que se organizan por su interés sobre el espacio del CU, especialmente sobre la reserva ViCCU, Club de Observación de Aves (COA) y Grupo de Ecología y Conservación del Ambiente (GECA), lo que reforzó la dinámica de intercambio entre pares, y dio lugar en el cierre a remarcar la importancia de contar con la voz de los involucrados, que generalmente disponen de un conocimiento profundo y único sobre el territorio. Más allá de este trabajo práctico concreto, del 2016 al 2018 las actividades de estos grupos - ViCCU, COA, GECA - y la incidencia de los estudiantes sobre el espacio de la reserva fueron aumentando, y se notó progresivamente en los talleres un mayor grado de conocimiento general sobre el espacio del CU, fuera de las experiencias particulares de algunas y algunos estudiantes más involucrados.

Los actores identificados en los distintos años se presentan en el cuadro 2. A la hora de identificar actores notamos que en algunos casos tales como Eventos en el Club River Plate y Comercios, bares, kioscos fue una dificultad visualizar a la/s persona/s que lo/s encarnan. También generó dudas si lo que se mapeaba eran las actividades que estos actores hacen como colectivos o también se debían identificar las actividades que desarrollan individualmente por intereses personales. Respecto a esto último, se aclaró que era difícil identificar a los actores fuera de sus roles cuando hacen uso del espacio de CU. 
Urdampilleta, C.; Borón, C. I.; Fischer, S.; Ithuralde, R. E.

Cuadro 2 - Actores identificados por año de cursada. Se exponen literalmente las categorías surgidas.

\begin{tabular}{|c|c|c|c|}
\hline Actores Identificados & 2016 & 2017 & 2018 \\
\hline Estudiantes & $\mathrm{X}$ & $\mathrm{X}$ & $\mathrm{X}$ \\
\hline Investigadores & $\mathrm{X}$ & & \multirow{2}{*}{$\mathrm{X}$} \\
\hline Docentes & $\mathrm{X}$ & & \\
\hline No Docentes / Gremio No docente & $\mathrm{X}$ & $\mathrm{X}$ & $\mathrm{X}$ \\
\hline Autoridades de las Facultades & $\mathrm{X}$ & $\mathrm{X}$ & $\mathrm{X}$ \\
\hline Rectorado UBA & & $\mathrm{X}$ & $\mathrm{X}$ \\
\hline Gobierno C.A.B.A. & $\mathrm{X}$ & & $\mathrm{X}$ \\
\hline Intendencia & $\mathrm{X}$ & & \\
\hline Policía / Seguridad Privada & $\mathrm{X}$ & $\mathrm{X}$ & $\mathrm{X}$ \\
\hline Usuarios campo de deportes & $\mathrm{X}$ & & \\
\hline Usuarios del Club Arquería y Club Universitario de Buenos Aires & & & $\mathrm{X}$ \\
\hline Eventos en Estadio "River Plate" & & $\mathrm{X}$ & \\
\hline Comercios, Bares, Kioscos & & $\mathrm{X}$ & \\
\hline Colectiveros & $\mathrm{X}$ & & \\
\hline Pescadores & $\mathrm{X}$ & $\mathrm{X}$ & $\mathrm{X}$ \\
\hline Interesados en asentarse en la zona / Asentamientos / Ocupas & $\mathrm{X}$ & $\mathrm{X}$ & $\mathrm{X}$ \\
\hline Vecinos & $\mathrm{X}$ & & \\
\hline Aldeanos / Eco-Aldea Velatropa & \multirow{2}{*}{$\mathrm{X}$} & $\mathrm{X}$ & \multirow{2}{*}{$\mathrm{X}$} \\
\hline Vivero Comunitario Ciudad Universitaria - ViCCU & & $\mathrm{X}$ & \\
\hline Club Observador de Aves - COA & \multirow{2}{*}{ ONGs } & $\mathrm{X}$ & \multirow{2}{*}{ ONGs } \\
\hline Grupo de Ecología y Conservación del Ambiente -GECA & & $\mathrm{X}$ & \\
\hline Total & 15 & 13 & 12 \\
\hline
\end{tabular}

Los actores que comparten una cruz fueron identificados como un único grupo en un año de cursada y como grupos distintos en otro año (ej. Investigadores y Docentes).

Fuente: Elaboración de los autores a partir del trabajo de las y los estudiantes en los talleres.

Se construyeron mapas representando los usos de cada actor identificado y un mapa unificado con todos los actores superpuestos. En la Figura 2 se presentan los mapas correspondientes a ONGs-2016 y Velatropa-ViCCU-2018 como ejemplos, y en la Figura 3, los mapas unificados de 2016 y 2018. 
Figura 2A - Mapa del uso e interés del CU de ONGs-2016. Figura 2B - Mapa del uso e interés del CU de "Velatropa-ViCCU-2018"
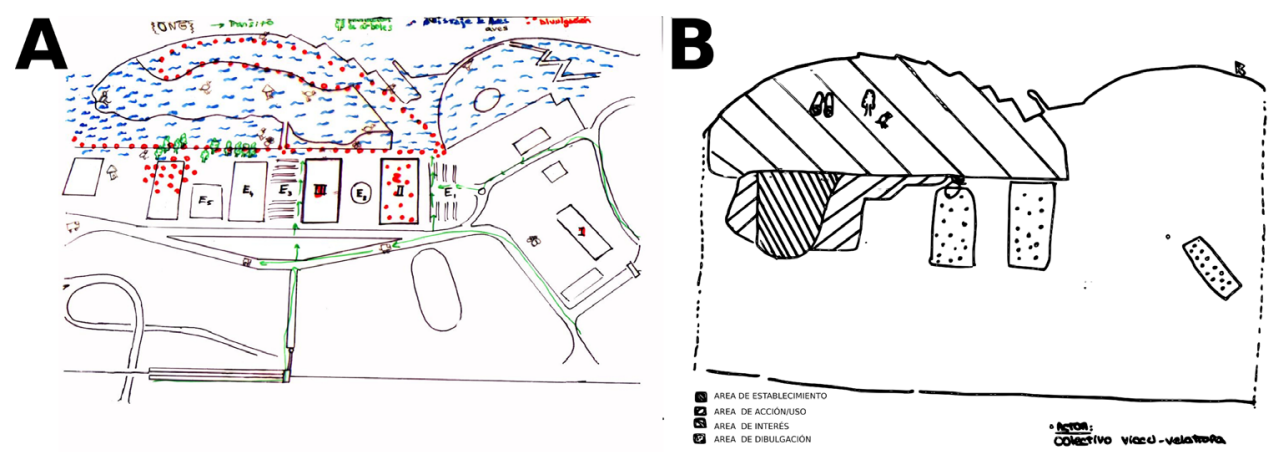

En (2A), los puntos indican actividades de difusión, las ondas, avistaje de aves, los árboles, plantación de árboles, y las flechas, el tránsito.

En (2B), las líneas densas corresponden al área de asentamiento, las de media densidad corresponden al área de acción/uso, las líneas menos densas, área de interés, y los puntos, al área de divulgación.

Fuente: Producción del estudiantado.

Figuras 3A y 3B - Mapas unificados de 2016 y 2018 respectivamente
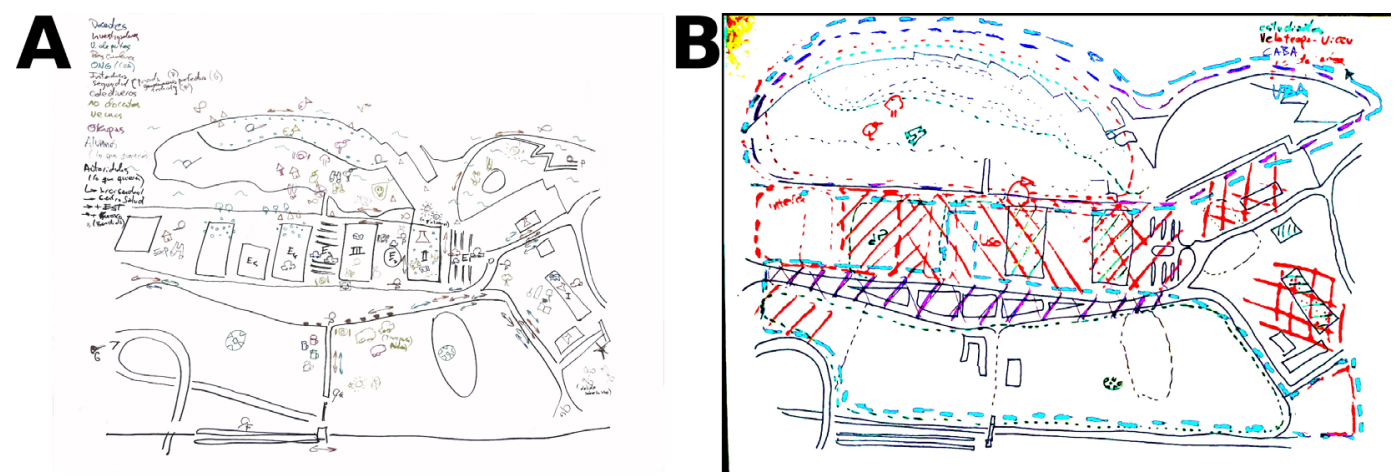

Los distintos colores representan diferentes actores.

Fuente: Producción propia a partir de las construcciones de los grupos de estudiantes.

La superposición de los mapas permitió visualizar dos situaciones contrastantes: áreas de uso normalizado con mucha superposición (edificios, áreas de tránsito, acceso y bar), donde se identifican pocos conflictos; y áreas verdes extensas con poca infraestructura y poca normalización del uso del espacio (área de la reserva, de la eco-aldea y del ViCCU), en donde se superponen usos muy diversos y visiones disímiles. Estas áreas son las que se corresponden con 
los conflictos reconocidos en la discusión sobre el mapa final pero no mapeados al considerar los actores por separado. Incluso en el último año se comenzaron dos obras de construcción de edificios que ocupan una buena parte del espacio entre los pabellones I, II y III, que hasta el momento era parquizado. Sin embargo, este último proceso no fue puesto en discusión por el estudiantado para sorpresa del grupo docente.

El mapeo permite visualizar en el espacio los intereses diferenciados por actores y cómo esos intereses se articulan pudiendo implicar conflictos explícitos, disputas silenciosas o coexistencias con distintos grados de acuerdo y diálogo. Se logra visibilizar también cómo los intereses de los actores de los espacios opera sobre la planificación y diseño del mismo; o cómo directamente el uso del mismo -sin una institucionalización- va formando el espacio y generando un paisaje particular.

Si bien la identificación de conflictos surgió principalmente en la discusión, ocupando un lugar preponderante en la puesta en común verbal, un número reducido de ellos fueron volcados en los mapas por actores. Estos últimos son los identificados con problemáticas materiales puntuales como la basura o contaminación. Las disputas por visiones contrapuestas sobre el uso del espacio entre actores no fueron representadas en los mapas individuales, sino que surgieron principalmente al completar la visión superpuesta de los actores. En todos los años se favoreció el intercambio entre las voces y conocimiento de las y los participantes. Esta dinámica, en todos los casos, propició un rico proceso de reconstrucción de la historia reciente de usos del espacio y de disputas del mismo.

Algunas de las preguntas y ejes que suscitaron intercambios entre las y los participantes en los distintos años fueron: la productividad de poner en cuestión las referencias - Norte/ Sur de los mapas - que nos facilitan el entendimiento de los mismos, la definición de conflictos socio-ambientales, qué disciplinas se encargan de realizar este tipo de estudios, la invisibilización de los sectores con necesidades habitacionales en relación a los actores con interés en establecer la reserva.

El Trabajo Práctico fue ampliamente aceptado por docentes y estudiantes. El estudiantado sugirió hacer ajustes en el tiempo, dado que algunas exposiciones les resultaban muy largas, e incluir moderadores para los momentos de debate, que propicien una mayor circulación de la palabra. En gran medida, apreciaron positivamente estudiar el propio espacio donde se asienta su facultad y expresaron desconocer en gran medida los actores principales que utilizan el predio de la Ciudad Universitaria, de qué forma lo hacen, las actividades que realizan y sus visiones sobre el espacio que habitan. Un tercio del estudiantado manifestó que quisieran tener más información y que el trabajo "depende de las interpretaciones", que "podría buscarse complementar esos aportes individuales con datos certeros sobre la situación legal-social-ambiental [aportar estudios hechos sobre la problemática del lugar o de otros que sirvan de referencia]" o "realizar una investigación seria antes de mapear", mostrando cierto apego hacia la objetividad propia (en la doxa hegemónica) de las ciencias naturales (BOURDIEU, 2003). El trabajo con las subjetividades de las y los actores intervinientes es escaso o nulo en el resto de la carrera, constituyendo este trabajo práctico un primer esfuerzo por ponerlas en primer plano de análisis.

El cuerpo docente resaltó la gran participación que hubo por parte de estudiantes durante el trabajo práctico. Coincidente con lo expresado por los y las estudiantes, los y las docentes observaron el conflicto generado por ellos y ellas por la dificultad de ser objetivos en el análisis de un espacio que usan a diario, para lo cual manifestaron la necesidad de un trabajo 
más preciso respecto a las y los actores y de profundizar en el trabajo de "identificar actores en relación a sus características, usos e intereses, y posteriormente categorizar y ver las relaciones entre ellos en función de un conflicto determinado".

Año a año se fueron realizando reuniones de evaluación y adaptación de la planificación del trabajo práctico para mejorar el desarrollo del taller. Desde el segundo año de realización, 2017, este esquema de trabajo práctico propuesto se enmarca en el primer tema del curso, conflictos socio-ambientales. Finalmente, se acordó la necesidad de incorporar instancias de evaluación sobre las temáticas abordadas en este trabajo práctico. Todas estas observaciones se han tomado en cuenta para realizar modificaciones para la siguiente cursada.

\section{Conclusiones}

Las dinámicas planteadas propiciaronla identificación de los distintos actores involucrados, visualización espacial de los usos que hacen del espacio, la identificación de diferentes interacciones y su problematización. Esta herramienta didáctica y técnica resultó un dispositivo valioso para generar apropiación de los conceptos teóricos socio-ambientales que aborda la materia, construir una mirada más amplia sobre el uso de los espacios en campus, además de colaborar con la construcción de un espacio de diálogo sobre el territorio que permite enriquecer la mirada sobre los diferentes actores y los intereses que persiguen. Resultó ante todo un fértil espacio para experimentar unas formas de hacer ciencia distintas a las que se enseñan mayoritariamente en la Facultad de Ciencias Exactas y Naturales (donde predomina un discurso de objetividad racional), en las que los significados producidos por los actores en los territorios forman parte (de forma consciente) de la práctica científica, y para debatir acerca de la importancia de estos puntos de vista contradictorios a la hora de analizar un conflicto ambiental.

\section{Agradecimientos}

A Marco Dreyer, integrante del Vivero Comunitario Ciudad Universitaria (ViCCU), por compartir sus conocimientos y por su participación fundamental. A las docentes de Ecología y Desarrollo, Carolina Facchinetti, Verónica Loetti y Haydée Pizarro por el espacio, la predisposición, el acompañamiento y el apoyo. A Mariana Totino por la revisión ortográfica y gramatical.

\section{Referencias}

ACEVEDO-DÍAZ, J. A.; VÁZQUEZ ALONSO, Á.; MANASSERO MAS, M. A. E1 movimiento ciencia-tecnología-sociedad y la enseñanza de las ciencias. [Madrid]: OEI: sala de lectura CTS+I, [2009]. Recuperado de: http://www.oei.es/historico/salactsi/ acevedo13.htm. Visitado en: 1 mayo 2018. 
BOURDIEU, P. E1 oficio de científico: ciencia de la ciencia y reflexividad. Barcelona: Anagrama, 2003.

BRENNER, L. Gobernanza ambiental, actores sociales y conflictos en las áreas naturales protegidas mexicanas. Revista Mexicana de Sociología, México, v. 72, n. 2, p. 283-310, 2010.

CENDALES GONZÁLEZ, L.; TORRES CARRILLO, A. La sistematización como experiencia investigativa y formativa. La Piragua: revista latinoamericana de educación política, Ciudad de Panamá, n. 23, p. 29-38, 2006. Recuperado de: http:/ / ceaal.org/images/ documentos/lapiragua23-1.pdf. Visitado en: 20 ago. 2019.

CENTRO INTERDISCIPLINARIO DE EXPERIMENTACIÓN Y EDUCACIÓN AMBIENTAL. Velatropa, 10 años. Buenos Aires: Ciudad Universitaria, 2017. Recuperado de: https://pt.scribd.com/document/366696926/Velatropa-10-Anos. Visitado en: 20 ago. 2019.

COARECN. Historia de la reserva ecológica Costanera Norte. Buenos Aires:

COARECN, 2015. Recuperado de: http:// coarecn.blogspot.com.ar/p/la-reserva.html. Visitado en: 8 feb. 2017.

DÍAZ, S.; QUÉTIER, F.; CÁCERES, D. M.; TRAINOR, S. F.; PÉREZHARGUINDEGUY, N.; BRET-HARTE, M. S.; PEÑA-CLAROS, M.; POORTER, L. Linking functional diversity and social actor strategies in a framework for interdisciplinary analysis of nature's benefits to society. Proceedings of the National Academy of Sciences, Washington, v. 3, n. 108, p. 895-902, 2011. DOI: https://doi.org/10.1073/ pnas. 1017993108.

FERNANDES, B. M. Sobre la tipología de los territorios. [Acción Tierra, 2009]. Recuperado de: http:/ /acciontierra.org/IMG/pdf/BERNARDO_TIPOLOGIA_DE_ TERRITORIOS_espanol.pdf. Visitado en: 26 mayo 2013.

GALEANO, E. H. Ser como ellos y otros artículos. Madrid: Siglo XXI, 1992.

GARCÍA, R. Interdisciplinariedad y sistemas complejos. In: CONFEDERACIÓN DE TRABAJADORES DE LA EDUCACIÓN DE LA REPÚBLICA ARGENTINA.

Educación en ambiente para el desarrollo sustentable. Buenos Aires: CTERA-EMV, 1999. p. 39-66.

GOOGLE earth. [2018]. Recuperado de: https://www.google.com/earth/. Visitado en: 18 oct. 2018.

HACE un mes se resistieron al desalojo: al final trasladaron a los habitantes de Villa

Gay. Clarín, Buenos Aires, 18 jul 1998. Recuperado de: http://edant.clarin.com/ diario/1998/07/18/e-06301d.htm. Visitado en: 8 feb. 2017.

HARVEY, D. El nuevo imperialismo. Madrid: Akal, 2004. 
LEFF, E. Complejidad, racionalidad ambiental y diálogo de saberes: hacia una pedagogía ambiental. Desenvolvimento e Meio Ambiente, Curitiba, v. 16, n. 1, p. 11-19, 2007. Recuperado de: https://revistas.ufpr.br/made/article/viewFile/11901/8397. Visitado en: 19 ago. 2019.

LEFF, E. La pedagogía del ambiente. In: CONFEDERACIÓN DE TRABAJADORES DE LA EDUCACIÓN DE LA REPÚBLICA ARGENTINA. Educación en ambiente para el desarrollo sustentable. Buenos Aires: CTERA-EMV, 1999. p. 9-14.

MANZANAL, M. Territorio, poder e instituciones: una perspectiva crítica sobre la producción del territorio. In: MANZANAL, M.; ARZENO, M.; NUSSBAUMER, B.

(coomp.). Territorios en construcción: actores, tramas y gobiernos, entre la cooperación y el conflicto. Buenos Aires: Fundación Centro Integral Comunicación, Cultura y Sociedad, 2007. p.15-50, 2007.

MARCOMINI, S. C.; LÓPEZ, R. A. Generación de nuevos ecosistemas litorales por albardones de relleno en la costa de la ciudad de Buenos Aires. Revista de la Asociación

Geológica Argentina, Buenos Aires, v. 59, n. 2, p. 261-72, 2004.

MASSARINI, A.; SCHNEK, A. (coord.). Ciencia entre todxs: tecnociencia en contexto social, una propuesta de enseñanza. Buenos Aires: Paidós, 2015.

QUIJANO, A.; WALLERSTEIN, I. M. La americanidad como concepto, o América en el moderno sistema mundial. Revista Internacional de Ciencias Sociales, Barcelona, v. 44, n. 4, p. 583-591, 1992. Recuperado de: https://unesdoc.unesco.org/ark:/48223/ pf0000092840_spa. Visitado en: 19 ago. 2019.

RISLER, J.; ARES, P. Manual de mapeo colectivo: recursos cartográficos críticos para procesos territoriales de creación colaborativa. Buenos Aires: Tinta Limón, 2013.

RISLER, J.; ARES, P. Talleres de mapeo: recursos lúdicos y visuales para la construcción de conocimiento colectivo. Ecología Política, Barcelona, n. 48, p. 28-32, 2014. Recuperado de: https://www.ecologiapolitica.info/novaweb2/?p=1918. Visitado en: 19 ago. 2019.

ROCHA L. Desalojaron una villa en Núñez. La Nación, Buenos Aires, 2006. Recuperado de: http://www.lanacion.com.ar/823235-desalojaron-una-villa-en-nunez. Visitado en: 8 feb. 2017.

RODRÍGUEZ, S. Escritos de Simón Rodríguez. Caracas: Imprenta Nacional, 1954.

SÁNCHEZ, R. Y.; PÉREZ, A. Mapeo 2.0: ampliando los límites de la cartografía crítica. Ecología Política, Barcelona, n. 48, p. 24-27, 2014.

SLETTO, B.; BRYAN, J.; TORRADO, M.; HALE, C; BARRY, D. Territorialidad, mapeo participativo y política sobre los recursos naturales: la experiencia de América Latina.

Cuadernos de Geografía: revista colombiana de geografía, Bogotá, n. 22, n. 2, p. 193-209, 2013. DOI: https://doi.org/10.15446/rcdg.v22n2.37014. 
TAPELLA, E. El mapeo de actores claves: versión perliminar. Córdoba: Universidad Nacional de Córdoba, [2007]. (Documento de trabajo del proyecto efectos de la biodiversidad funcional sobre procesos ecosistémicos, servicios ecosistémicos y sustentabilidad en las Américas). Recuperado de: https://planificacionsocialunsj.files. wordpress.com/2011/09/quc3a9-es-el-mapeo-de-actores-tapella1.pdf. Visitado en: 30 mar. 2018.

UNIVERSIDAD DE BUENOS AIRES. Facultad de Ciencias Exactas y Naturales. Resolución de Consejo Directivo no 2767/14 y no 141/15. [2015] Recuperado de: http://2007-2016.exactas.uba.ar/institucional/display.php?estructura=1\&desarrollo=0\&id_ caja=311\&nivel. Visitado en: 8 feb. 2017.

UNIVERSIDAD DE BUENOS AIRES. Facultad de Ciencias Exactas y Naturales. Departamento de Ecología, Genética y Evolución. Materias del área Ecología 1er cuat: ecología y desarrollo. [2017]. Recuperado de: http://www.ege.fcen.uba.ar/academico/ materias-de-grado/materias-del-ciclo-superior-primer-cuatrimestre/materias-del-areaecologia/ecologia-y-desarrollo. Visitado en: 4 mar. 2018.

ZEMMELMAN, H. Conocimiento y sujetos sociales: contribuciones al estudio del presente. La Paz: Instituto Internacional de Integración del Convenio Andrés Bello, 2011. 